\title{
Reversal reactions in the skin lesions of AMFES patients: incidence and risk factors
}

\author{
PAUL SAUNDERSON*, SHIBRU GEBRE* \& \\ PETER BYASS** \\ *ALERT, PO Box 165, Addis Ababa, Ethiopia \\ **School of Community Health Science, Nottingham University, \\ Nottingham, UK and Department of Public Health and Clinical \\ Medicine, Umeå University, Umeå, Sweden
}

Accepted for publication 30 June 2000

Summary Reversal reactions affect the skin and/or nerves of leprosy patients. This paper looks at reversal reactions involving the skin in 594 new patients in central Ethiopia, followed for between 6 and 11 years after the start of treatment. The incidence of reversal reaction declines steadily after the start of treatment, but the first episode may occur as long as 5 years after diagnosis in both paucibacillary (PB) and multibacillary $(\mathrm{MB})$ patients. Recurrent episodes occurred up to 6 years after diagnosis. PB patients were at greatest risk for reversal reaction in the first year after diagnosis and MB patients in the first 4 years. The highest incidence rate was 18 episodes per 100 person years in MB patients during the first year after diagnosis. The ratio of the incidence rates for the first 3 years in MB versus PB patients is 2.4 (95\% CI 1.6-3.8). This study confirms that starting effective treatment and borderline classification are risk factors for reversal reactions. Pregnancy/delivery in the 6 months prior to diagnosis was a significant risk factor for presenting with a reversal reaction [relative risk (RR) 5.9 (95\% CI 2.1-16.5)], but later pregnancies were not associated with an increased risk. Being female was a significant risk factor for the late appearance of the first episode of reversal reaction. Having a reversal reaction in the first year after diagnosis was a highly significant risk factor for the development of later reactions [RR in PB cases 11.9 (95\% CI 3.4-41.7); in MB cases 6.4 (95\% CI 3.8-10.6)]. Being HIV positive was a risk factor for developing recurrent reversal reactions, although only three out of 29 recurrent cases were HIV positive [RR 2.7 (95\% CI 1.4-5.1)].

\section{Introduction}

With the success of multi-drug therapy in the treatment of leprosy, attention has focused on the problem of leprosy reactions, which are now the most significant issue in the management

Correspondence to: P. Saunderson, ALM, 1 ALM Way, Greenville, SC 29601, USA (e-mail: psaunderson@ leprosy.org) 
of the individual patient. ${ }^{1}$ Much is known of the epidemiology of reactions, but their incidence after the period of MDT is less well documented because of the lack of longterm, prospective studies. ${ }^{2}$ The range of clinical presentations and their management has been well documented, especially for reversal, or type 1 , reactions. ${ }^{3}$

Clinically, in reversal reactions the leprosy lesions become erythematous, raised oedematous and infiltrated; there may be oedema of the hands and/or feet. Lesions occasionally ulcerate and new lesions may appear, presumably due to inflammation around inapparent foci of bacilli. ${ }^{3}$ Corticosteroids are the drug of choice in severe reversal reactions with nerve involvement, while simple analgesics are effective in mild reactions.

Reversal reactions are characterized by episodes of increased inflammatory activity in skin and/or nerves of patients with borderline leprosy. ${ }^{2}$ In practice, there are two main clinical presentations, in which either skin signs or neurological signs predominate. Reversal reactions often present with typical signs and symptoms of inflammation in the skin lesions. There may or may not be associated nerve damage, but the pain in the skin lesions causes the patient to seek help or the inflamed lesions may be noticed by the health worker at a routine clinic visit. In a second group of patients, skin signs are less obvious or are even absent, and nerve function impairment is the sign that inflammation is present in the nerves. ${ }^{4}$ The patient may complain of nerve tenderness or loss of function, but often the damage occurs insidiously and is only noted by the health worker through routine nerve function tests.

Risk factors for reversal reactions have been looked at in a number of contexts, but as yet there is no method of confidently predicting which patients are at risk. ${ }^{2,5}$ In the literature it is often difficult to distinguish risk factors for neuritis from those for reversal (or type 1) reactions. However, several risk factors have been documented, including bactericidal drug regimens, ${ }^{6}$ attending as a self-reporting case,${ }^{7}$ having a facial patch, as a risk for lagophthalmos, ${ }^{5,8}$ the presence of anti PGL- 1 antibodies and a positive lepromin test, ${ }^{5,9}$ during MDT and the subsequent 6 months, ${ }^{10,11}$ extensive disease, indicated by the number of body areas involved, ${ }^{5,11}$ borderline classification, ${ }^{11}$ BCG vaccination ${ }^{2}$ and a positive BI. ${ }^{5}$

This paper examines the incidence of, and risk factors for, reversal reactions affecting the skin, following van Brakel, ${ }^{11}$ while an accompanying paper looks at the much more complex issue of nerve involvement in some detail. In this paper, therefore, the term reversal reaction implies signs of inflammation in leprosy skin lesions, although nerve involvement may also be present.

\section{Materials and methods}

A prospective cohort study of leprosy patients treated with fixed-duration MDT, was set up at the All Africa Leprosy, Tuberculosis and Rehabilitation Centre (ALERT) in central Ethiopia in 1988, with the objectives of determining the incidence rates of relapse, reactions and nerve damage, and the risk factors for these events. In all, 660 patients were enrolled in this ALERT MDT Field Evaluation Study, known as AMFES, between March 1988 and March 1993. ${ }^{12,13}$ Ten patients were excluded soon after diagnosis, either because the diagnosis was changed or the enrolment procedures were incorrectly followed. A further 56 patients, who were relapses after dapsone monotherapy, were not included in this review of reversal reactions; this group includes four of the $22 \mathrm{HIV}$-positive individuals in the cohort. Five hundred and ninety-four new cases are reviewed. After completion of treatment, the following proportions attended 
for follow-up: $92 \%$ attended for the first year, $87 \%$ the second, $76 \%$ the third, $71 \%$ the forth and $62 \%$ the fifth.

Cases were classified as $\mathrm{MB}$ if they were classified clinically as $\mathrm{BB}, \mathrm{BL}$ or LL in the Ridley-Jopling classification. In addition, BT patients were classified as MB if they had a positive skin smear at any site, although three BT patients with a bacillary index (BI) of 1 were treated as paucibacillary cases in the first year of the study, under earlier guidelines. BT patients with many skin lesions were classified as PB if their smears were negative.

A reversal reaction could be diagnosed by field staff on the basis of the clinical finding of signs of inflammation in leprosy skin lesions. ${ }^{13}$ Findings may include complaints by the patient of pain and/or tenderness in the skin lesions, swelling and warmth of the lesions, and sometimes erythema. Nerve function impairment was assessed and recorded separately. Recurrent reversal reactions were defined as further episodes occurring more than 3 months after the start of a previous episode, whatever treatment may have been given. The term therefore includes those whose reaction flared up again immediately steroids were stopped, and those in whom a subsequent reaction occurred months or years later. Episodes of reaction may have been observed at a routine follow-up examination, or the patient could easily attend as a self-reporting case.

While type 1 or reversal reactions can occur years after the start of treatment, they are much more common at the start of treatment and in the first year thereafter. The experience of patients is therefore analysed by year after diagnosis. This allows the quantification of the change in incidence over time. Risk factors are analysed in a similar way, allowing the characterization of specific risk factors for late reactions.

Longitudinal patient records within this prospective cohort study were managed throughout using dBase software. Analysis used Epi-Info software and logistic regression modelling of multiplicative relative risks was performed using Egret.

\section{Results}

PREVALENCE AT DIAGNOSIS

Table 1 indicates the prevalence of various events at the time of diagnosis. Reversal reactions were present in $5 \%$ of new cases (4.3\% of MB cases and $5.8 \%$ of PB cases).

The following possible risk factors were examined for association with the presence of reversal reaction at diagnosis: age, sex, classification, BI, contact status, HIV status, lepromin status, pregnancy (either pregnant at the time of diagnosis, delivery in the previous 6 months

Table 1. The frequency of various complications present at diagnosis. Numbers of cases and (percentages)

\begin{tabular}{lccc}
\hline & $\begin{array}{c}\text { Number of } \\
\text { cases }\end{array}$ & $\begin{array}{c}\text { Reversal reaction } \\
\text { alone }\end{array}$ & $\begin{array}{c}\text { Reversal reaction }+ \\
\text { acute neuritis }\end{array}$ \\
\hline MB cases & 300 & $1(0.3 \%)$ & $12(4 \%)$ \\
PB cases & 294 & $2(0.7 \%)$ & $15(5 \%)$ \\
Totals & 594 & $3(0.5 \%)$ & $27(4.5 \%)$ \\
\hline
\end{tabular}


or in the previous 3 years) and delay in presentation. The only factor significantly associated with an increased risk of reversal reaction at diagnosis was the delivery of a baby in the 6 months prior to diagnosis: relative risk (RR) 5.9 (95\% CI 2.1-16.5). These results were essentially the same in both univariate and multivariate analyses.

RISK FACTORS FOR, AND TIMING OF, THE FIRST REVERSAL REACTION AFTER DIAGNOSIS

Ninety-eight of the 594 cases $(16.5 \%)$ under review developed a reversal reaction at some time, including those with a reaction at presentation. This represents $11 \%$ of PB cases and $22 \%$ of MB cases. Twenty-nine of the 98 cases (29\%) had more than one episode of reversal reaction.

Table 2 shows the risk factors for developing a reversal reaction, while Figure 1 shows the

Table 2. Risk factors for the development of a reversal reaction. (reaction cases $=98$; study population $=594$; cases included in the multivariate analysis $=509$, with 86 reaction cases). Note: the stepwise multivariate analysis only includes those factors whose association with the dependant variable approaches significance

\begin{tabular}{|c|c|c|c|}
\hline Factor & $\begin{array}{l}\text { Number of cases } \\
\text { with reversal } \\
\text { reaction }\end{array}$ & $\begin{array}{c}\text { Univariate analysis } \\
\text { of relative risk } \\
(95 \% \mathrm{CI})\end{array}$ & $\begin{array}{l}\text { Stepwise multivariate } \\
\text { analysis of relative } \\
\text { risk }(95 \% \mathrm{CI})\end{array}$ \\
\hline PB & $32 / 294$ & 1.0 & \\
\hline MB & $66 / 300$ & $2.3(1.5-3.7)$ & - \\
\hline Classification: other & $8 / 93$ & 1.0 & \\
\hline Classification: borderline & $90 / 501$ & $2.3(1.1-5.0)$ & $\begin{array}{c}4.2(1.8-9.8) \\
P<0.001\end{array}$ \\
\hline Negative skin smear & $34 / 312$ & 1.0 & \\
\hline Positive skin smear & $61 / 268$ & $2.4(1.5-3.9)$ & $\begin{array}{c}3.6(2.2-6.1) \\
P<0.001\end{array}$ \\
\hline Male & $55 / 378$ & 1.0 & \\
\hline Female & $43 / 216$ & $1.5(0.9-2.3)$ & $\begin{array}{c}1.6(1.0-2.7) \\
P=0.51\end{array}$ \\
\hline No pregnancy & $85 / 545$ & 1.0 & \\
\hline Any pregnancy since 3 & $13 / 49$ & $1.9(1.0-3.8)$ & - \\
\hline Age $<20$ & $32 / 177$ & 1.0 & \\
\hline Age 20-49 & $49 / 316$ & $0.8(0.5-1.4)$ & - \\
\hline Age $50+$ & $17 / 101$ & $0.9(0.5-1.7)$ & - \\
\hline HIV negative & $85 / 507$ & 1.0 & \\
\hline HIV positive & $4 / 18$ & $1.4(0.4-4.8)$ & - \\
\hline Lepromin negative & $29 / 117$ & 1.0 & - \\
\hline Lepromin positive & $20 / 89$ & $0.9(0.4-1.8)$ & - \\
\hline No history of contact & $71 / 434$ & 1.0 & \\
\hline Contact history positive & $27 / 160$ & $1.0(0.6-1.7)$ & - \\
\hline Delay $<4$ years & $80 / 464$ & 1.0 & \\
\hline Delay $>4$ years & $18 / 124$ & $0.8(0.5-1.2)$ & - \\
\hline
\end{tabular}




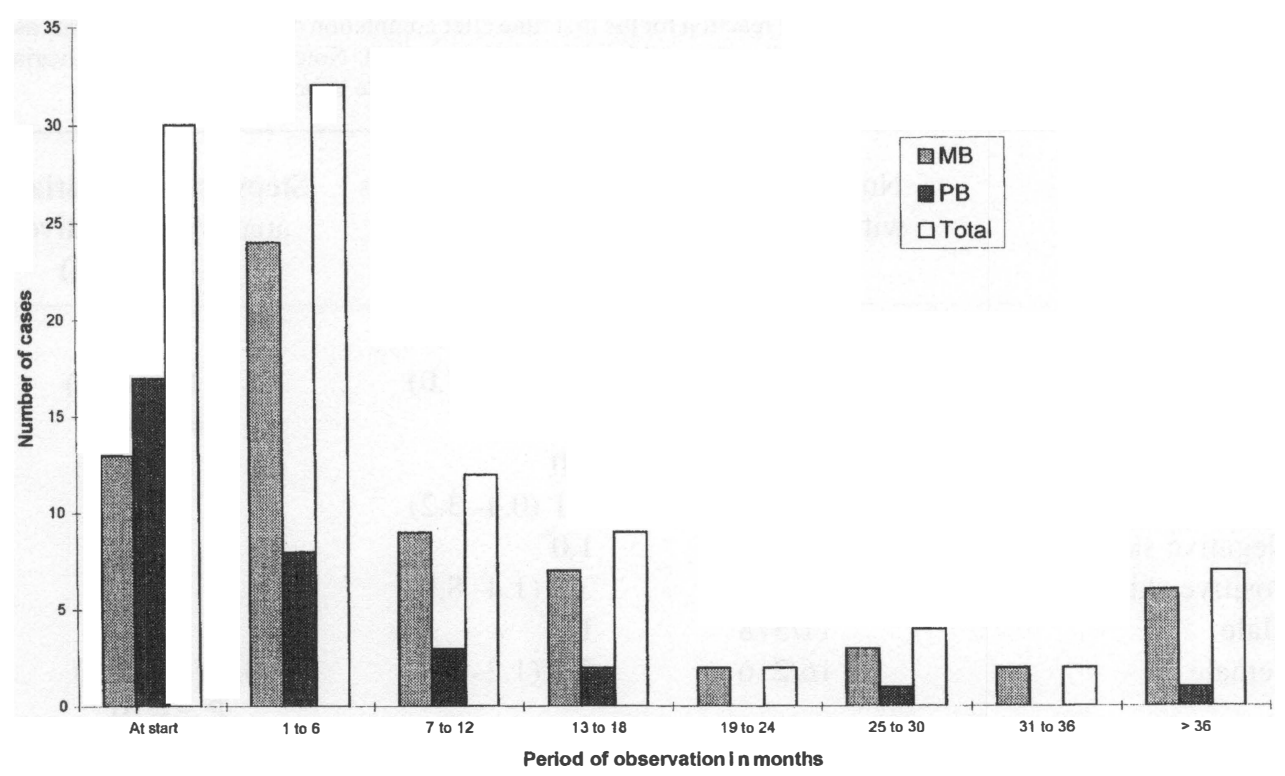

Figure 1. Timing of first episode of reversal reaction $(n=98)$.

timing of the first episode of reversal reaction in 98 cases. Borderline classification and a positive skin smear at diagnosis were significant risk factors for reversal reactions, while being female approached significance as a risk factor.

While reversal reactions can occasionally occur for the first time more than 5 years after the start of treatment (this occurred in two MB patients in this cohort), the majority of first episodes occur in the first year. Twenty-eight of $32 \mathrm{~PB}$ patients $(88 \%)$ and 46 of $66 \mathrm{MB}$ patients $(70 \%)$ had their first episode within the first year after starting treatment. Twenty-five of the PB patients had their first episode within the first 6 months.

Possible risk factors for being amongst the seven $\mathrm{PB}$ patients whose first reversal reaction occurred after the 6-month period of MDT, were examined. No association was found with any of the following factors: sex, age, HIV status, delay in presentation, pregnancy, contact status and lepromin status. Patients with WHO Impairment Grade 2 at diagnosis had an increased risk of developing their first reversal reaction after the end of MDT (RR 2.34; 95\% CI 1.2-4.6), although the fact that they already have impairment strongly suggests that they have had a reactional episode prior to diagnosis.

The same risk factors were examined for the $20 \mathrm{MB}$ patients whose first reversal reaction occurred later than 1 year after the start of treatment, i.e. after the currently recommended period of MDT. There was no association with any of these factors (including the WHO Impairment Grade at diagnosis), except being female (RR 4.01; 95\% CI 1.65-9.72), but this was not associated with later pregnancies. The BI at diagnosis was not associated with late reversal reaction in $\mathrm{MB}$ patients.

Table 3 gives the risk factors for developing a reversal reaction after the end of MDT for $\mathrm{PB}$ and $\mathrm{MB}$ cases together. Being female and being multibacillary are the only statistically significant risk factors, while pregnancy is not an important factor in this cohort. The multivariate analysis was repeated for females alone, but pregnancy did not become an important risk factor in that regression model either (RR 1.3). 
Table 3. Risk factors for developing a reversal reaction for the first time after completion of MDT. (late reaction cases $=27$; study population $=594$; cases included in the multivariate analysis $=509$ ). Note: the stepwise multivariate analysis only includes those factors whose association with the dependant variable approaches significance

\begin{tabular}{|c|c|c|c|}
\hline Factor & $\begin{array}{l}\text { Number of cases } \\
\text { with late reversal } \\
\text { reaction }\end{array}$ & $\begin{array}{l}\text { Univariate analysis } \\
\text { of relative risk } \\
(95 \% \mathrm{CI})\end{array}$ & $\begin{array}{l}\text { Stepwise multivariate } \\
\text { analysis of relative } \\
\text { risk }(95 \% \mathrm{CI})\end{array}$ \\
\hline PB & $7 / 294$ & 1.0 & \\
\hline MB & $20 / 300$ & $2.9(1.2-7.0)$ & $\begin{array}{c}3.7(1.4-10.4) \\
P=0.006\end{array}$ \\
\hline Classification: other & $4 / 93$ & 1.0 & \\
\hline Classification: borderline & $23 / 501$ & $1.1(0.4-3.2)$ & - \\
\hline Negative skin smear & $7 / 312$ & 1.0 & \\
\hline Positive skin smear & $19 / 268$ & $3.3(1.4-8.0)$ & - \\
\hline Male & $11 / 378$ & 1.0 & \\
\hline Female & $16 / 216$ & $2.7(1.2-5.9)$ & $\begin{array}{c}3.4(1.4-8.2) \\
P=0.01\end{array}$ \\
\hline No pregnancy & $23 / 545$ & 1.0 & \\
\hline $\begin{array}{l}\text { Any pregnancy since } 3 \\
\text { years before diagnosis }\end{array}$ & $4 / 49$ & $2.0(0.7-6.1)$ & - \\
\hline Age $<20$ & $11 / 177$ & 1.0 & \\
\hline Age 20-49 & $12 / 316$ & $0.6(0.3-1.4)$ & - \\
\hline Age $50+$ & $4 / 101$ & $0.6(0.2-2.0)$ & - \\
\hline HIV negative & $22 / 507$ & 1.0 & \\
\hline HIV positive & $2 / 18$ & $2.8(0.6-12)$ & - \\
\hline Lepromin negative & $12 / 117$ & 1.0 & - \\
\hline Lepromin positive & $4 / 89$ & $0.4(0.1-1.3)$ & - \\
\hline No leprosy contact & $17 / 434$ & 1.0 & \\
\hline Contact history positive & $10 / 160$ & $1.6(0.7-3.7)$ & - \\
\hline Delay $<4$ years & $21 / 464$ & 1.0 & \\
\hline Delay $>4$ years & $6 / 124$ & $0.9(0.5-1.6)$ & - \\
\hline
\end{tabular}

INCIDENCE OF REVERSAL REACTIONS BY YEAR

Figure 2 shows the incidence rate of reversal reactions by year for both MB and PB cases. Seventeen patients (nine $\mathrm{PB}$ and eight $\mathrm{MB}$ ) had reversal reactions only at the start, so are not included here. Eighty-one patients (23 PB and $58 \mathrm{MB}$ ) had 130 episodes of reversal reaction (36 in PB cases and 94 in MB cases) after starting treatment.

For the first 3 years taken together, the overall incidence of reversal reactions is 7.8 per 100 person-years-at-risk (PYAR) (95\% CI 6.2-9.3). For MB cases the figures are 10.6 PYAR (95\% CI 8.2-13.0) and for PB cases the figures are 4.3 PYAR (95\% CI 2.6-6.0). The rate ratio is 2.4 (95\% CI 1.6-3.8).

RECURRENT REVERSAL REACTIONS

For PB cases there were nine episodes after the first year and of these four were first episodes 


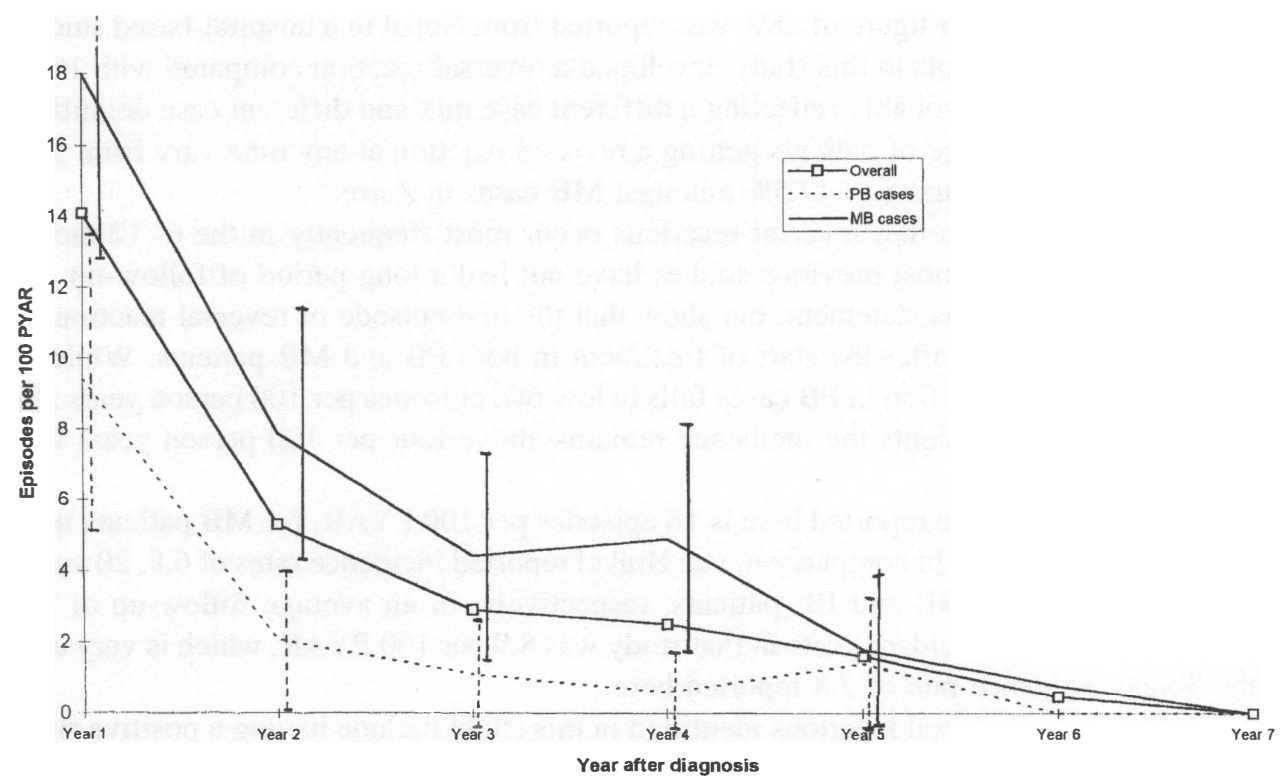

Figure 2. Incidence rate of reversal reactions after diagnosis, by year (130 episodes in 98 patients). $95 \%$ confidence intervals are given for $\mathrm{MB}$ and $\mathrm{PB}$ cases.

and five were repeat episodes. Having a reversal reaction at the start or during the first year is associated with an increased risk of having another episode later than 1 year after diagnosis (RR 11.9; 95\% CI 3.4-41.7).

For MB cases there were 43 episodes after the first year, including 20 first episodes and 23 repeat episodes. There is an increased risk of a late reversal reaction if an episode occurred at the start or during the first year (RR 6.35; 95\% CI 3.8-10.6).

Other risk factors for recurrent reversal reaction were looked for. There was no association with age, sex, classification, BI, contact status, lepromin status, pregnancy, impairment status at start or at RFT, or delay in presentation. Being HIV positive was associated with an increased risk of having more than one episode of reversal reaction in the new cases examined here (RR: 2.7 ; 95\% CI 1.4-5.1), although the number of cases involved is small - of the 29 patients with recurrent reversal reactions, two had no HIV test result, three were positive and 24 were negative; of the four HIV-positive patients who had any reversal reaction, however, three had recurrent episodes.

\section{Discussion}

The definition of reversal reaction still remains a problem and different studies use different definitions; some include episodes of neuritis or nerve function impairment (NFI) within a broad definition of reversal reaction. ${ }^{2}$ In this report, reversal reaction refers only to the clinical syndrome in which leprosy skin lesions show signs of inflammation, whether or not there was accompanying NFI. A separate report examines the data for NFI.

Previous reports indicate a prevalence of reversal reaction at the time of diagnosis of between $2.6 \%$ and $6.4 \%$, and the findings presented here are compatible with those figures, ${ }^{2}$ 
although the much higher figure of $28 \%$ was reported from Nepal in a hospital-based study. ${ }^{11}$ In all, $16.5 \%$ of all patients in this study developed a reversal reaction compared with $10.9 \%$ in Hyderabad, India, ${ }^{14}$ probably reflecting a different case mix and different case definitions. Figures for the percentage of patients getting a reversal reaction at any time vary from $3.5 \%$ amongst PB cases in Malawi to $47.5 \%$ amongst MB cases in Zaire. ${ }^{2}$

Although it is known that reversal reactions occur most frequently in the 6-12 months after starting MDT, ${ }^{3,10}$ most previous studies have not had a long period of follow-up. The AMFES data confirm this statement, but show that the first episode of reversal reaction can occur as late as 5 years after the start of treatment in both PB and MB patients. While the incidence of reversal reaction in PB cases falls below two episodes per 100 person years after the first year, in MB patients the incidence remains above four per 100 person years for 4 years.

The highest incidence reported here is 18 episodes per 100 PYAR, for MB patients in the first year after diagnosis. In comparison, van Brakel reported incidence rates of 6.8, 20 and 15 per 100 PYAR in BT, BB and BL patients, respectively, in an average follow-up of 20.7 months. ${ }^{11}$ The overall incidence rate in that study was 8.9 per 100 PYAR, which is very close to the 3-year incidence rate of 7.8 reported here.

Risk factors for reversal reactions identified in this study include having a positive smear at diagnosis and borderline classification. Unfortunately, information about which patches developed a reaction was not recorded, so it is not possible to examine the importance of a reacting face patch as a risk factor for other complications. Female sex as a possible risk factor, independently of pregnancy, just fails to reach significance. Interestingly, the previously quoted study in Nepal found that amongst BT cases, females had a significantly greater risk of developing a reversal reaction, although data for pregnancies were not available. $^{11}$

Pregnancy and lactation are reported to be risk factors for reversal reactions, but the association has not been quantified and remains unclear. ${ }^{2,15}$ Amongst the AMFES women, delivery of a baby in the 6 months before the diagnosis of leprosy was made, was associated with an increased risk of reversal reaction at diagnosis. Later pregnancies were fewer in number and were not associated with reversal reactions, but being female was associated with the late appearance of the first reversal reaction.

Recurrent episodes of reversal reaction are an important phenomenon, as they may be associated with continuing nerve damage and a gradual deterioration in the patient's condition. Reactions have previously been reported up to 5 or 6 years after diagnosis. ${ }^{10,14}$ A reaction in the first year after diagnosis gave an increased risk of a later reaction and being HIV positive, while not associated with an increased risk of reversal reaction as such, was associated with recurrent episodes in those who had reversal reactions, the result just reaching significance at the 5\% level. In an analysis of all the HIV-tested patients in the AMFES cohort, including those in the group who were relapses after dapsone monotherapy, a similar result was found, although that result just failed to reach significance. ${ }^{16}$

In conclusion, borderline leprosy is the major risk factor for developing a reversal reaction, while the major period of risk is the 12 months after the start of treatment for paucibacillary patients. In multibacillary patients the period of risk for first and repeat episodes lasts about 4 years from the start of treatment. Later episodes occur in only very few patients. HIV infection increases the risk of recurrent reactions. Pregnancy and lactation are shown to be risk factors at the time of diagnosis, but being female also seems to be of some importance as a risk factor, being associated with late first reactions in this study. 


\section{Acknowledgements}

We thank the staff of the ALERT Leprosy/TB Control Division for their dedication and perseverance in managing the patients and collecting data over so many years. The financial support of ILEP, through Netherlands Leprosy Relief (NLR), has been constant throughout the 12 years of the study and is gratefully acknowledged. We also thank ALERT as a whole for institutional and administrative support.

\section{References}

1 Britton WJ. The management of leprosy reversal reactions (Editorial). Lepr Rev, 1998; 69: 225-234.

2 Lienhardt C, Fine PEM. Type 1 reaction, neuritis and disability in leprosy. What is the current epidemiological situation? Lepr Rev, 1994; 65: 9-33.

${ }^{3}$ Rose P, Waters MF. Reversal reactions in leprosy and their management (Editorial). Lepr Rev, 1991; 62: 113-121.

${ }^{4}$ van Brakel WH, Khawas IB. Silent neuropathy in leprosy: an epidemiological description. Lepr Rev, 1994; 65: 350-360.

${ }^{5}$ Roche PW, Le Master J, Butlin CR. Risk factors for type 1 reactions in leprosy. Int J Lepr, 1997; 65: 450-455.

${ }^{6}$ Groenen G, Janssens L, Kayembe T et al. Prospective study on the relationship between intensive bactericidal therapy and leprosy reactions. Int J Lepr, 1986; 54: 236-244.

7 Boerrigter G, Ponnighaus JM, Fine PEM. Preliminary appraisal of a WHO-recommended multiple drug regimen in paucibacillary leprosy patients in Malawi. Int J Lepr, 1988; 56: 406-417.

${ }^{8}$ Hogeweg M, Kiran KU, Suneetha S. The significance of facial patches and type I reaction for the development of facial nerve damage in leprosy. A retrospective study among 1226 paucibacillary leprosy patients. Lepr Rev, 1991; 62: 143-149.

9 Roche PW, Theuvenet WJ, Britton WJ. Risk factors for type-1 reactions in borderline leprosy patients. Lancet, 1991; 338: 654-657.

10 Becx-Bleumink M, Berhe D. Occurrence of reactions, their diagnosis and management in leprosy patients treated with multidrug therapy; experience in the leprosy control program of the All Africa Leprosy and Rehabilitation Training Center (ALERT) in Ethiopia. Int J Lepr, 1992; 60: 173-184.

11 van Brakel WH, Khawas IB, Lucas SB. Reactions in leprosy: an epidemiological study of 386 patients in west Nepal. Lepr Rev, 1994; 65: 190-203.

12 de Rijk AJ, Gebre S, Byass P, Berhanu T. Field evaluation of WHO-MDT of fixed duration at ALERT, Ethiopia: the AMFES project - I. MDT course completion, case-holding and another score for disability grading. Lepr Rev, 1994; 65: 305-319.

13 de Rijk AJ, Gebre S, Byass P, Berhanu T. Field evaluation of WHO-MDT of fixed duration, at ALERT, Ethiopia: the AMFES project, part 2. Reactions and neuritis during and after MDT in PB and MB leprosy patients. Lepr Rev, 1994; 65: 320-332.

14 Lockwood DNJ, Vinayakumar S, Stanley JN et al. Clinical features and outcome of reversal (type 1) reactions in Hyderabad, India. Int J Lepr, 1993; 61: 8-15.

15 Lockwood DNJ, Sinha HH. Pregnancy and leprosy: a comprehensive literature review. Int J Lepr, 1999; 67: 6-12.

16 Gebre S, Saunderson PR, Messele T, Byass P. The effect of HIV status on the clinical picture of leprosy: a prospective study in Ethiopia. Lepr Rev, 2000; 71: 338-343. 